\title{
A-16
}

\section{EFECTO DE LA FRECUENCIA DE RIEGO SOBRE LA CALIDAD DEL TOMATE EN LA REGIÓN COSTERA DEL NORDESTE BRASILEÑO}

\author{
Morais, G.M. ${ }^{2}(P)$, Rebouças Neto, M. O. ${ }^{1}$, Brasil, S. O. S ${ }^{3}$, Palau, C.V. ${ }^{4}$, \\ Vasconcelos, D.V.
}

\author{
${ }^{1}$ Profesor, IFPI - Campus Corrente, Correntes - PI, agromario@gmail.com \\ ${ }^{2}$ Doctoranda en Ingeniería Agrícola, UFC, Fortaleza - CE, gislane_mendez@yahoo.com.br \\ ${ }^{3}$ Estudiante de grado en Ingeniería Agronómica, UFC, Fortaleza - CE, \\ suane_brasil@hotmail.com \\ ${ }^{4}$ PDI, Universitat Politècnica de València, Dpto. Ingeniería Rural, virpaes@agf.upv.es \\ ${ }^{5}$ Doctora en Ingeniería Agrícola, Dpto. de Ingeniería Agrícola, UFC, Fortaleza - Ceará, \\ denisevasconcelos@hotmail.com
}

\section{Resumen}

El objetivo de este estudio fue evaluar la calidad de la fruta de los tomates para diferentes frecuencias de riego en dos años de cultivo en la región costera del nordeste brasileño. El experimento fue conducido durante el período de agosto a diciembre de 2011 y 2012 en el área experimental de la Estación Meteorológica de la Universidad Federal del Ceará, situada en la ciudad Fortaleza, nordeste brasileño. El sistema de riego utilizado fue localizado con goteros integrados de caudal nominal $4 \mathrm{~L} \mathrm{~h}^{-1}$. El diseño experimental fue con bloques al azar factorial de 6x4. Los tratamientos adoptados fueron: F2 (dosis de riego cada dos días), F3 (dosis de riego cada tres días), F4 (dosis de riego cada cuatro días), F5 (dosis de riego cada cinco días), F6 (dosis de riego cada seis días) y F7 (dosis de riego a cada siete días). La dosis de riego diaria se estimó mediante un balance de agua entre evapotranspiración del cultivo y precipitaciones efectivas en la zona de estudio. Esta dosis aplicada será acumulativa en función de la frecuencia de riego de cada prueba experimental. Al final del experimento, las variables analizadas fueron: la firmeza de la fruta (FF), los sólidos solubles (SS), la acidez titulable (AT), el pH y la relación SS / AT. El manejo del riego aplicado al cultivo del tomate ha demostrado ser beneficioso por sus efectos positivos en la mejora de la calidad de los frutos para la industria de transformación. Los resultados que comparan diferentes frecuencias de riego dieron información útil sobre la posibilidad de mejorar su calidad nutricional. El intervalo de riego de 7 días proporcionó frutos de mejor calidad para el procesamiento industrial, y un intervalo de riego menor proporcionó frutos con características óptimas para su consumo en fresco.

\section{1- Introducción y objetivos}

Pocas son las hortalizas que a nivel mundial presentan una demanda tan alta como el tomate. Del grupo de las hortalizas, el tomate es la especie más importante, tanto desde una perspectiva social como desde un punto de vista económico, por el volumen de producción y de creación de empleos.

Según los datos de FAOSTAT (Food and Agriculture Organization of the United Nations), la producción mundial de tomates en 2013 fue de 163 millones de toneladas. Brasil es uno de los mayores productores, ocupando el octavo lugar seguido por España. La producción brasileña fue de cerca de 4 millones de toneladas en una superficie de 
60.000 hectáreas. Aproximadamente el $24 \%$ de la producción nacional se destina a la industria de transformación y el resto para consumo en fresco.

La frecuencia de riego requerida por un cultivo en un clima específico depende del agua que se puede almacenar en un determinado suelo después de un riego, de la profundidad efectiva del sistema radicular, del porcentaje de superficie cubierta y de la precipitación de la zona. En las regiones semiáridas, en general, las plantas requieren más agua por ciclo (Andrade y Borges Junior, 2008).

Dentro de las hortalizas, el tomate es uno de losmás exigentes en agua con un consumo superior a la media. En el fruto maduro del tomate, el contenido en agua es cercano al 93\% e su constitución (Santana et al., 2011). Las necesidades totales de agua al año, después de trasplantar el cultivo de tomate son de 400-600 mm, dependiendo del clima (Silva et al., 2000). Según Oliveira et al. (2011) un exceso o deficit de agua es uno de los factores más limitantes para la obtención de altos rendimientos. La influencia de las prácticas de riego sobre la calidad de los frutos de tomate no ha sido suficientemente estudiada.

En particular, existen numerosos estudios sobre los efectos de la cantidad de riego en el cultivo del tomate que consideran la calidad de los frutos sólo en términos de sólidos solubles y de rendimiento (Hanson \& May, 2004; Hanson et al., 2006; Machado et al., 2005; Zegbe-Dominguez et al., 2003). Sin embargo, hay poca literatura sobre la influencia de la frecuencia de riego en el valor nutricional de tomate (Mahajan y Singh, 2006).

Siendo así, el objetivo de este trabajo fue identificar la frecuencia de riego que puede proporcionar una mayor productividad y una mejor calidad en los frutos de tomate para su procesamiento en la industria en las condiciones agroclimáticas de la región costera del nordeste brasileño.

\section{2- Materiales y métodos}

El experimento fue conducido durante el período de agosto a diciembre de 2011 y 2012, en el área experimental de la Estación Meteorológica de la Universidad Federal del Ceará, situada en la ciudad Fortaleza, nordeste brasileño, a $3^{\circ} 44^{\prime}$ de latitud sur, 38 $33^{\circ}$ ' de longitud oeste y a 19,5 m de altitud. En esta región, el clima es tropical lluvioso, con precipitaciones importantes en el verano-otoño. Los datos mensuales de las variables climáticas recogidas durante los experimentos se muestran en la Tabla 1.

Tabla 1. Los datos mensuales de las variables climáticas durante la realización de experimentos, Fortaleza, Ceará, 2011 y 2012.

\begin{tabular}{lcccccc}
\hline \multirow{2}{*}{ Mes } & \multicolumn{2}{c}{ Temperatura del aire $\left({ }^{\circ} \mathrm{C}\right)$} & \multicolumn{2}{c}{ Precipitación $(\mathrm{mm})$} & \multicolumn{2}{c}{ Humedad Relativa (\%) } \\
\cline { 2 - 7 } & 2011 & 2012 & 2011 & 2012 & 2011 & 2012 \\
\hline Agosto & 26,6 & 28,7 & 51,0 & 0,0 & 71,0 & 73,3 \\
Septiembre & 27,0 & 28,8 & 0,0 & 0,0 & 67,0 & 73,2 \\
Octubre & 27,1 & 28,9 & 23,8 & 2,3 & 72 & 72,9 \\
Noviembre & 27,4 & 29,3 & 7,3 & 0,5 & 73 & 73,3 \\
Diciembre & 27,7 & 29,7 & 6,7 & 1,8 & 71 & 74,1 \\
\hline
\end{tabular}

Fuente: Estación agrometeorológica de la Universidad Federal de Ceará.

El sistema de riego utilizado fue localizado con goteros integrados de caudal nominal $4 \mathrm{~L} \mathrm{~h}^{-1}$. El diseño experimental fue de bloques al azar factorial de $6 \times 4$. Los tratamientos 
adoptados fueron: F2 (dosis de riego cada dos días), F3 (dosis de riego cada tres días), F4 (dosis de riego cada cuatro días), F5 (dosis de riego cada cinco días), F6 (dosis de riego cada seis días) y F7 (dosis de riego a cada siete días).

Para los experimentos, se utilizó el tomate caqui (Lycopersicon esculentum, Mill), híbrido F1 de la empresa Topseed, que tiene forma de crecimiento indeterminado.

La dosis de riego diaria se estimó mediante un balance de agua entre evapotranspiración del cultivo y precipitaciones efectivas en la zona de estudio. Esta dosis aplicada será acumulativa en función de la frecuencia de riego de cada prueba experimental. ecuación 1.

El tiempo de riego empleado en el experimento se determinó de acuerdo con la

$\mathrm{Ti}$ - tiempo de riego $(\mathrm{h})$;

$\mathrm{Di}$ - Dosis de riego $\left(\mathrm{mm} \mathrm{d}^{-1}\right)$;

$\mathrm{El}$ - espaciamiento entre las filas $(m)$;

Eg - espaciamiento entre goteros $(m)$;

Fc - factor de cobertura del suelo (sin dimensiones);

$\mathrm{Ei}$ - eficiencia de riego (sin dimensiones);

$\mathrm{q}_{\mathrm{g}}$ - caudal del gotero $\left(\mathrm{L} \mathrm{h}^{-1}\right)$.

La dosis del riego (Di) se estimó por ETo de Penman-Monteith, obtenida con los datos de una estación meteorológica automatizada equipados con sensores y accesorios necesarios para calcularla por el método de Penman-Monteith (Allen et al., 1998).

La siembra se realizó manualmente en bandejas de 128 celdas el de 15 julio 2011 y el 17 de julio de 2012, después de 26 días de la siembra se realizó el trasplante en campo con espacios de 1,0 y 0,5 m entre plantas de la misma fila. En el vigésimo día después del trasplante (DDT), cuando las plantas ya se habían aclimatado en el campo, se iniciaron los tratamientos.

En la fertilización de las plantas se utilizó urea y cloruro de potasio, correspondiente a $11,2 \mathrm{~g}$ por planta; lo que corresponde a las dosis de 300 y $400 \mathrm{~kg} \mathrm{ha}^{-1}$ de $\mathrm{N}$ y $\mathrm{K}_{2} \mathrm{O}_{2}$, respectivamente. El fósforo se aplicó todo de una vez con una dosis correspondiente a 200 $\mathrm{kg} \mathrm{ha}^{-1}$ de $\mathrm{P}_{2} \mathrm{O}_{5}$, los $2 / 3$ restantes de $\mathrm{N}$ y 11 aplicaciones parceladas de $\mathrm{K}_{2} \mathrm{O}$ a través de fertirrigación.

La cosecha se realizó del 70 al 90 DDT, donde se recogieron manualmente los frutos. Después de cosechar los frutos se colocaron en bolsas de plástico en cajas y fueron transportadas al Laboratorio de Fisiología y Tecnología de Postcosecha en EMBRAPA, las variables analizadas fueron: la firmeza de la fruta (FF), los sólidos solubles (SS), la acidez titulable (AT), el pH y la relación SS / AT.

Los resultados fueron sometidos a un análisis de regresión con un nivel de significación del $5 \%$ probando modelos: lineal, logarítmico, exponencial y polinómial cuadrático. 
La ecuación que mejor resultados proporcionó, se seleccionó como base en el análisis de los parámetros de regresión. Los análisis estadísticos se realizaron con la ayuda de las aplicaciones de Microsoft Office Excel (2007), y por medio de software ASSISTAT 7.6.

\section{3- Resultados y discusión}

De acuerdo al análisis de varianza, presentado en la tabla 2, las diferentes frecuencias de riego aplicadas influyen significativamente en la firmeza de la fruta (FF), los sólidos solubles (SS), la acidez titulable (AT), y la relación SS / AT en los dos años de experimentos, el nivel de significación del 1 y $5 \%$ en la prueba $\mathrm{F}$. Sólo el pH no mostró diferencias significativas.

Tabla 2. Resumen del análisis de varianza para firmeza media del fruto (FMF), sólidos solubles (SS), pH, acidez titulable (AT) y relación SS/AT, en plantas de tomate con diferentes frecuencias de riego.

\begin{tabular}{|c|c|c|c|c|c|c|}
\hline \multirow{3}{*}{ Fuente de variación } & \multirow{3}{*}{ GL } & \multicolumn{5}{|c|}{ Promedio } \\
\hline & & \multicolumn{5}{|c|}{ Agosto-Diciembre, 2011} \\
\hline & & FMF & SS & $\mathrm{pH}$ & AT & SS/AT \\
\hline Tratamientos & 5 & $25.89 *$ & $0.074^{*}$ & $0.023^{\mathrm{ns}}$ & $0.011^{\star \star}$ & $21.13^{\star \star}$ \\
\hline Bloques & 3 & $4.99^{\mathrm{ns}}$ & $0.002^{\mathrm{ns}}$ & $0.046^{*}$ & $0.00043^{\text {ns }}$ & $3.41^{\text {ns }}$ \\
\hline Residuo & 15 & 6.45 & 0.018 & 0.012 & 0.00108 & 2.74 \\
\hline \multirow[t]{3}{*}{$\mathrm{CV}(\%)$} & - & 17.26 & 3.26 & 2.26 & 9.09 & 13.36 \\
\hline & & \multicolumn{5}{|c|}{ Promedio } \\
\hline & & \multicolumn{5}{|c|}{ Agosto-Diciembre, 2012} \\
\hline Tratamientos & 5 & $21.77^{\star \star}$ & $1.153^{\star \star}$ & $0.020^{\mathrm{ns}}$ & $0.012^{* \star}$ & 45.54 *夫 \\
\hline Bloques & 3 & $0.562^{\text {ns }}$ & $0.223^{\text {ns }}$ & $0.018^{\text {ns }}$ & $0.00106^{\text {ns }}$ & $1.65^{\text {ns }}$ \\
\hline Residuo & 15 & 0.86 & 0.109 & 0.048 & 0.00150 & 4.25 \\
\hline CV(\%) & - & 6.23 & 6.37 & 4.51 & 10.52 & 14.11 \\
\hline
\end{tabular}

$\mathrm{FV}=$ Fuente de variación, $\mathrm{GL}=$ grado de libertad, $\mathrm{CV}=$ coeficiente de variación; $T=$ Testigo, ** $=$ Significativo a $1 \%,{ }^{*}$ Significativo a $5 \%$ y ${ }^{\text {ns }}=$ no significativo.

Para la variable firmeza de la fruta (Figura 1), el gráfico presenta una tendencia creciente lineal con el aumento de frecuencias de riego. La firmeza y la cantidad de agua son los principales atributos que determinan la calidad del almacenamiento de los frutos de tomate (Batu, 2004.; Dorais et al, 2001.; Viskelis et al., 2008). Cuanto mayor es la firmeza de la fruta mayor es suduración en el estante, debido a que la fruta es menos susceptible a los daños mecánicos durante el transporte. 


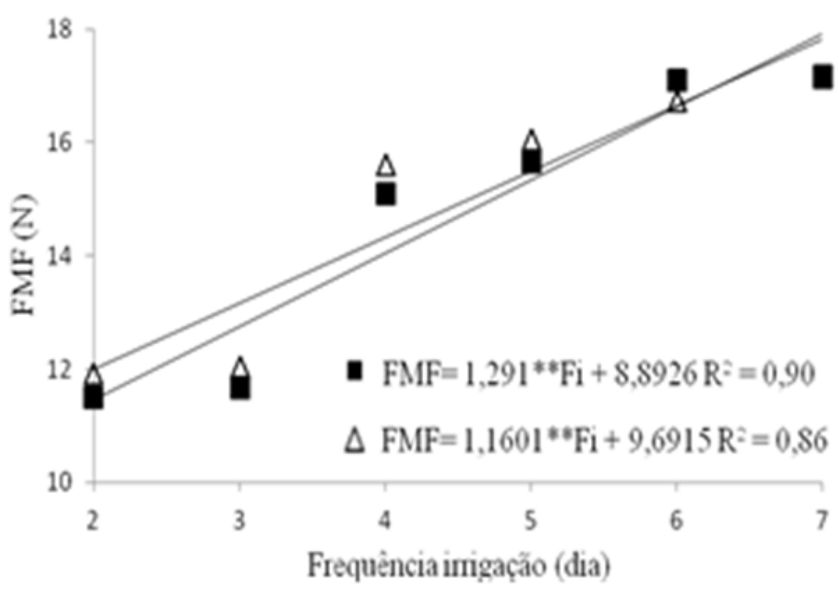

Figura 1. Firmeza media de los frutos de tomate en función de la frecuencia de riego, Fortaleza, Ceará, (ם) 2011 y $(\Delta)$ 2012. ${ }^{*}$ Significativo con un test t de Student, a 1\% de probabilidad.

Las frecuencias de riego afectan significativamente a los sólidos solubles (SS) entre los años 2011 y 2012 (Figura 2). El valor medio de SS en los dos años fue de 4,0 y 4,5 Brix, respectivamente, en la prueba con menor frecuencia (F2) y de 4,8 y 6,1 ${ }^{\circ}$ Brix para la frecuencia más amplia de aplicación de agua (F7). Estas diferencias entre los valores de sólidos solubles entre los años de estudiofue debida a lamayor precipitación durante 2011 en comparación con 2012, lo que puede haber causado menor cantidad de SS tanto para la frecuencia más baja como para la mayor, puesto que en el año de mayor precipitación las plantas sufrieron un estrés hídrico menor (Hanson \& Mai, 2004.; Hanson et al, 2006).

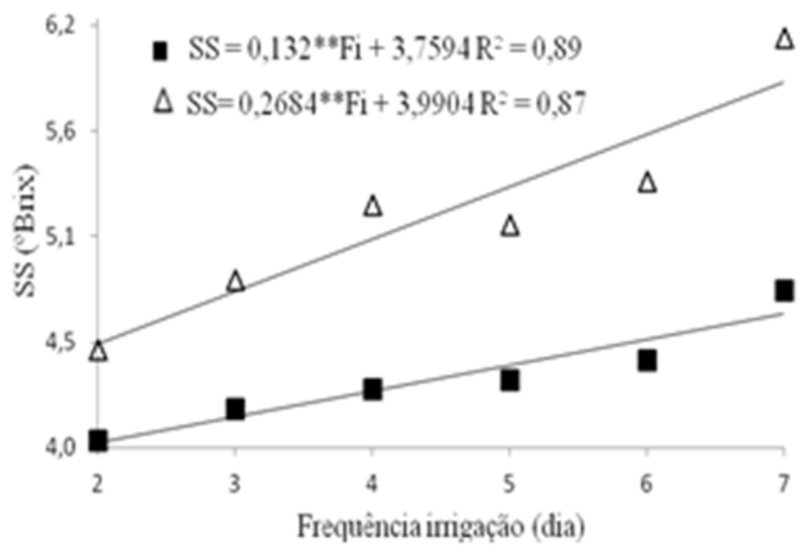

Figura 2. Sólidos solubles del fruto del tomate em función de la frecuencia de riego, Fortaleza, Ceará, (ロ 2011 y $(\triangle)$ 2012. ${ }^{\star}{ }^{\star}$ Significativo con un test t de Student, a 1\% de probabilidad.

Para la acidez titulable (Figura 3), los valores fueron de 0,451\% y 0,456\% para la mayor frecuencia de riego y de $0,320 \%$ y $0,319 \%$ para la menor frecuencia de riego, en los años 2011 y 2012, respectivamente. La acidez titulable de tomate para transformación industrial es por lo general cercana al 0,35\% (Garcia \& Barret, 2006).En nuestro estudio, los 
tomates con la frecuencia más baja de riego han producido frutos con un valor de acidez adecuado para la transformación industrial.

En particular, el aumento de intervalo de riego originó un incremento en la acidez titulable en ambos años. La verdad es que los valores de acidez titulable disminuyen y aumentan con la disponibilidad de agua. Algunos autores consideran que las concentraciones más altas de ácido en la fruta son resultado de un déficit de agua (Mitchell et al 1991; Tuzel et al., 1994).

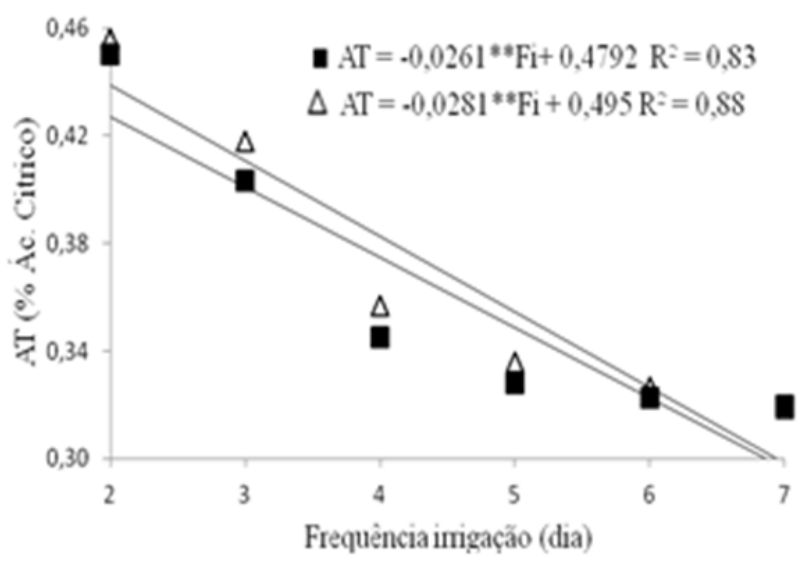

Figura 3. Acidez titulable de frutos de tomate en función de la frecuencia de riego, Fortaleza, Ceará, $(\square) 2011$ e $(\Delta)$ 2012. **Significativo con un test t de Student, a 1\% de probabilidad.

Para la relación sólidos solubles y acidez tilulable (Figura 4) se mostró un aumento lineal de esta relación con el aumento en el intervalo de riego, como se ha visto antes, esto se debe al aumento en sólidos solubles ocasionado por el estrés hídrico, mientras que la acidez titulable se reduce.

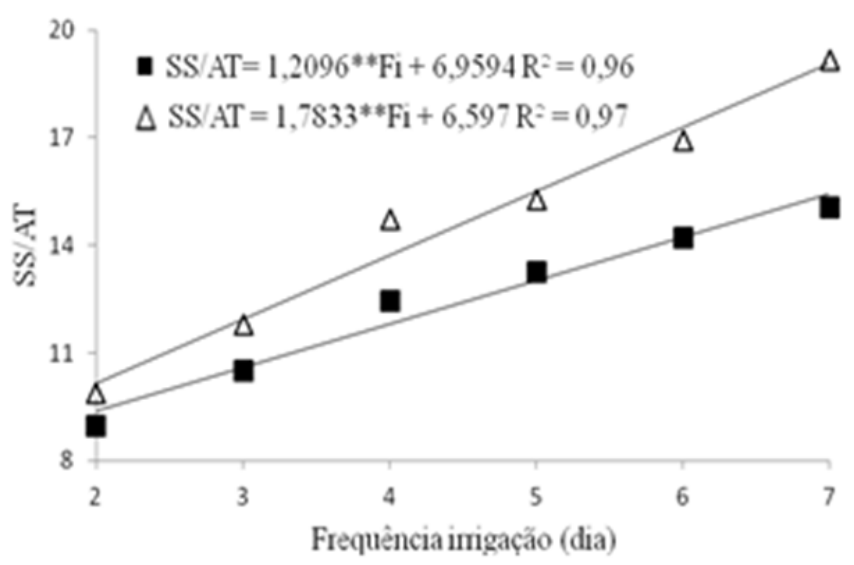

Figura 4. Relación entre sólidos solubles y acidez titulable en frutos de tomate en función de la frecuencia de riego, Fortaleza, Ceará, (⿴) 2011 e $(\triangle)$ 2012. **Significativo con un test $t$ de Student, a $1 \%$ de probabilidad. 
Los valores medios más altos fueron de 15 y 19,2, mientras que los mínimos fueron 9 y 9,9 obtenidos con el máximo y mínimo intervalo de riego en el año 2011 y 2012. El valor alto en esta relación indica una excelente combinación de azúcar y ácido que se correlaciona con un sabor suave, mientras que los valores bajos dan un sabor ácido al fruto.

Se observa que los valores encontrados en el año 2011 son menores que en 2012 y esa diferencia puede atribuirse a la variable climática de precipitación como se ha comentado anteriormente.

\section{4- Conclusiones}

El manejo del riego aplicado al cultivo del tomate ha demostrado ser beneficioso por sus efectos positivos en la mejora de la calidad de los frutos para la industria de transformación.

Los resultados que comparan diferentes frecuencias de riego dieron información útil sobre la posibilidad de mejorar su calidad nutricional. El intervalo de riego de 7 días proporcionó frutos de mejor calidad para el procesamiento industrial, y un intervalo de riego menor proporcionó frutos con características óptimas para su consumo en fresco.

\section{5- Agradecimientos}

Agradezco al Universidad Federal de Ceará - Brasil, por permitir el desarrollo de la investigación y el CNPq por la financiación.

\section{6- Bibliografia}

Allen, R. G., Pereira, L. S., Raes, D., Smith, M. (1998). Crop evapotranspiration - Guidelines for computing crop water requirements. Rome: FAO, FAO Irrigation and Drainage. Paper 56. 319p.

Andrade, c. L. T.; Borges Júnior, j. C. F. (2008). Uso e manejo de irrigação. 1ed. Brasília: Ed. EMBRAPA, 528p.

Batu, A. (2004). Determination of acceptable firmness and colour values of tomatoes. Journal of Food Engineering, 61, 471-475.

Dorais, M.; Papadopoulos, A.; Gosselin, A. (2001). Greenhouse tomato fruit quality. Horticultural Reviews, 26, 239-319.

Garcia E., and D.M. Barrett. Feb. 2006. Evaluation of Processing Tomatoes from Two Consecutive Growing Seasons: Quality Attributes, Peelability and Yield. Journal of Food Processing and Preservation, 30(1), 20-36.

Hanson, B.R.; May, D. M. (2004). Effect of subsurface drip irrigation on processing tomato yield, water table depth, soil salinity, and profitability. Agric. Water Manage, 68, 1-6.

Hanson, B. R.; Hutmacher, R. B.; May, D. M. (2006). Drip irrigation of tomato and cotton under shallow saline ground water conditions. Irrig. Drain. Sys. 20, 155-175. 
Oliveira, E. C.; Carvalho, J. A.; Rezende, F. C.; Freitas, W. A. (2011). Viabilidade técnica e econômica da produção de ervilha (Pisum sativum L.) cultivada sob diferentes lâminas de irrigação. Engenharia Agrícola, 31 (2), 324-333.

Machado, R. M. A.; Rosário, M.; Oliveira, G. (2005). Tomato root distribution, yield and fruit quality under different subsurface drip irrigation regimes and depths. Irrig. Sci. 24, 15-24.

Mahajan, G.; Singh, K.G. (2006). Response of greenhouse tomato to irrigation and fertigation. Agric. Water Manage. 84, 202-206.

Mitchell, J.P., Shennan, C., Grattan, S.R. (1991). Developmental changes in tomato fruit composition in response to water deficit and salinity. Plant Physiol. 83, 177-185.

Santana, M. J; Pereira, U. C; Beirigo, J. D. C; Souza, S. S; Campos, T. M; Vieira, T. A. (2011). Coeficientes de cultura para o tomateiro irrigado. Revista irriga, Botucatu, 16 (1) 1120.

Silva, J. B. C.; Giordano, L. B. (2000). Tomate para processamento industrial. Brasília: Embrapa Comunicação para Transferência de Tecnologia - Embrapa Hortaliças, 168p.

Tuzel, I.H., UI, M.A., Tuzel, Y. (1994). Effects of different irrigation intervals and rates on spring season glasshouse tomato production. II. Fruit quality. Acta Hort. 366, 389-396.

Viskelis, P.; Jankauskiene, J.; Bobinaite, R. (2008). Content of carotenoids and physical properties of tomatoes harvested at different ripening stages. In: FOODBALT, 166-170. 